Slide 1

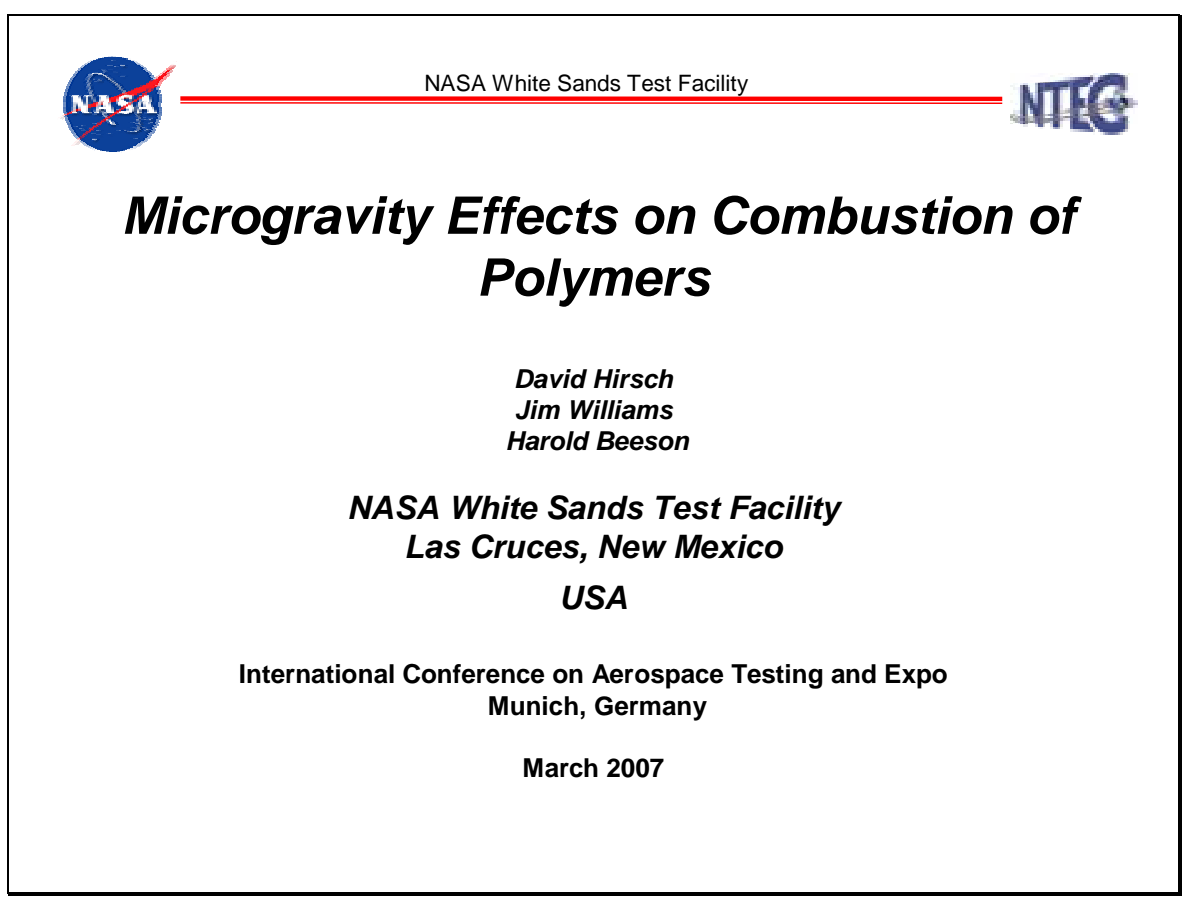

No notes for this slide.

Slide 2

\begin{tabular}{|c|c|}
\hline \multirow{2}{*}{\multicolumn{2}{|c|}{$\begin{array}{l}\text { NASA White Sands Test Facility } \\
\text { Topics }\end{array}$}} \\
\hline & \\
\hline \multicolumn{2}{|c|}{$\begin{array}{l}\text { - Major combustion processes and controlling } \\
\text { mechanisms in normal and microgravity } \\
\text { environments }\end{array}$} \\
\hline \multicolumn{2}{|c|}{$\begin{array}{l}\text { - Review of some buoyancy effects on combustion: } \\
\text { melting of thermoplastics; flame strength, geometry } \\
\text { and temperature; smoldering combustion }\end{array}$} \\
\hline \multirow{2}{*}{\multicolumn{2}{|c|}{$\begin{array}{l}\text { - Video comparing polymeric rods burning in normal } \\
\text { and microgravity environments } \\
\text { - Relation to spacecraft fire safety of current } \\
\text { knowledge of polymers' microgravity combustion }\end{array}$}} \\
\hline & \\
\hline March 2007 & $\begin{array}{l}\text { Hilisch, Williams, and Beeson on Microrgavity Effects on Combustion } \\
\text { of Polymers }\end{array}$ \\
\hline
\end{tabular}

No notes for this slide. 


\section{Slide 3}

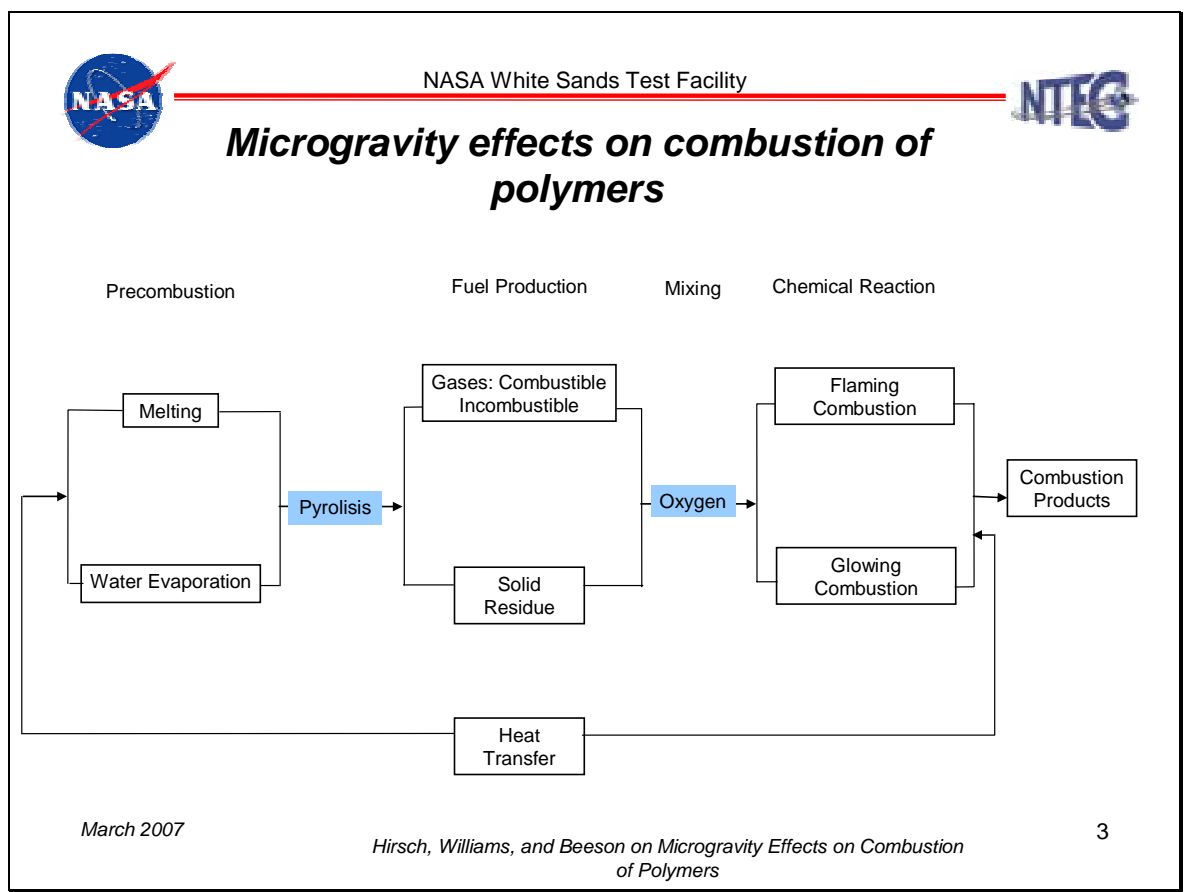

The major processes involved in combustion are similar both in normal normal gravity and microgravity. However, the absence of buoyancy may result in different controlling mechanisms of some processes.

1. Pre-combustion physical processes include melting and water evaporation. Further exposure to heat decomposes the polymers by pyrolisis; combustion gases or solids may result. Mixing with oxygen could result in a flammable mixture and the combustion may be initiated by an open flame, or the material may self-ignite. For most polymers, flaming combustion would result in the gas phase, while smoldering combustion could be a possibility for porous materials burning in the solid phase. The heat feedback to the material produces further melting and pyrolisis and if the heat generated exceeds the heat lost, the combustion would be self-sustained. 


\section{Slide 4}

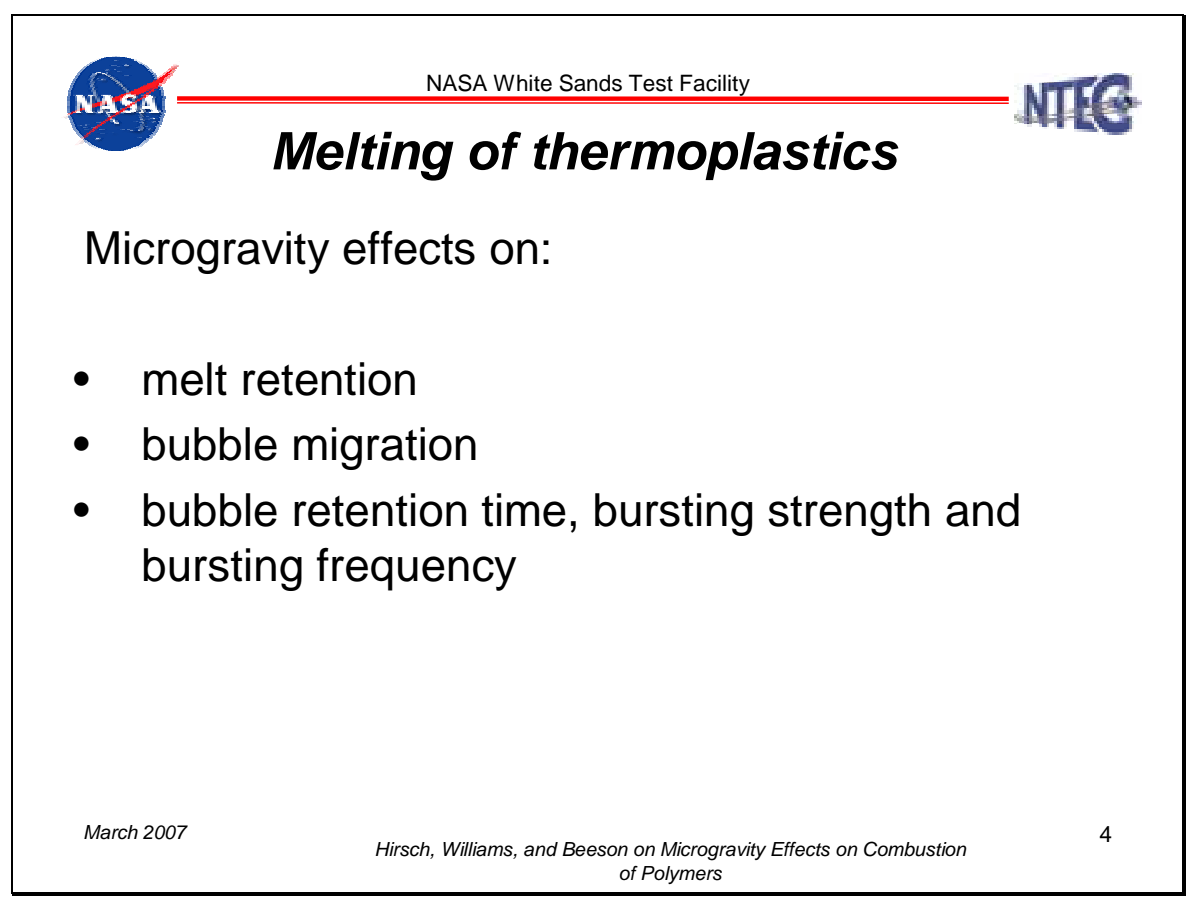

2. Thermoplastics are polymers which typically melt and this results in some major differences between the normal and microgravity conditions. As a matter of fact, thermoplastics present a fire hazard unique to microgravity. Reduced gravity results in melt retention; furthermore, during combustion, chemical reactions within the bulk of the material may generate internal bubbles. When the local temperature at a bubble nucleation site exceeds the degradation temperature of the polymer, bubble growth is initiated.

A temperature gradient induces migration of the bubble toward the regions of higher temperature by thermocapillary motion or viscosity gradients. Thermocapillary motion is driven by surface tension along the surface of the bubble, which create a tangential stress that causes fluid motion inside and outside the bubble. Neverthe-less, viscosity gradients appear to be a more realistic explanation of bubble migration, especially if the bubble growth rate is much larger than the translation speed.

Once at the surface, the bubble may be retained or burst through, depending on the internal pressure, growth rate, and the viscosity, surface tension, and viscoelasticity of the melt at the surface. Burning fuel vapor and occasionally molten fuel may be forcibly ejected, potentially spreading the fire in random directions, a phenomena which may be enhanced in microgravity. Lower flame temperatures in microgravity are associated with a lower melt temperature and consequently higher viscosity. The bubble may be retained longer at the melt surface, enhancing it's size and burst strength. Kashiwagi and Ohlemiller observed that for burning PMMA the bubble frequency increased and bursting was less violent with increasing levels of oxygen. 


\section{Slide 5}

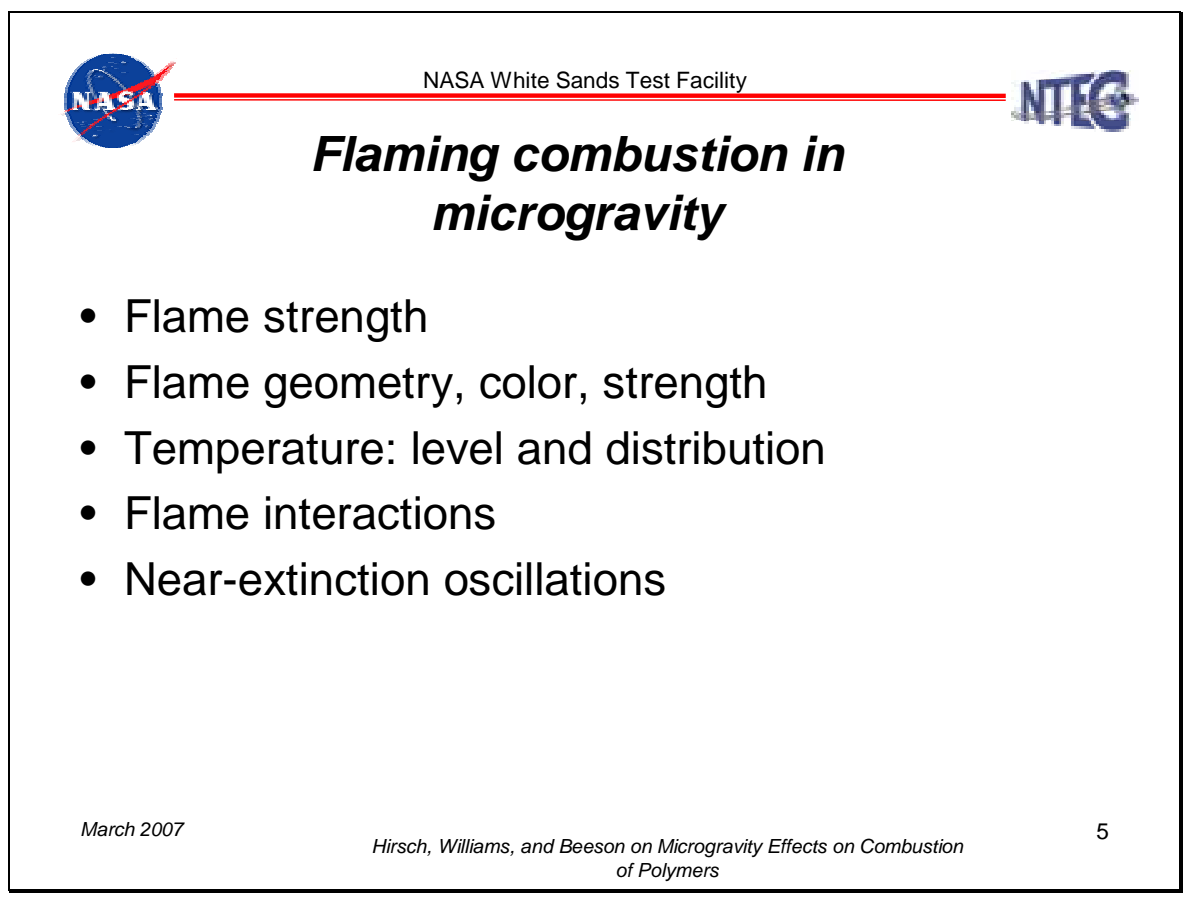

4. The visible flame in quiescent microgravity is different from that in normal gravity in a number of aspects:

A. Shape. For a rod material configuration, the microgravity flame is more spherical, with a reduced height-to-width ratio. Consequently the flame provides a large portion of the heat feedback to the burning region, which is different in normal gravity where only some of the fuel burns in the material vicinity, the rest being swept away and reacting in the plume region. There is a larger flame standoff in microgravity, indicating that the total heat feedback is less.

B. Color. The microgravity flames tend to be blue and not yellow (sooty). The reason is the reduced flame temperature in microgravity. The soot would be formed only at $1500 \mathrm{~F}$ or above (Glassman). The reduced flame temperature results from the fact that the heat loss relative to the heat generation rate is larger in microgravity even though the heat loss in microgravity is smaller. Never-the-less,radiative heat loss can be significant in microgravity and lead to quenched extinction. The radiative losses can be from the surface, from gas-phase species such as water and carbon dioxide, or from a combination of both.

C. The flame structure is different. For downward flame propagation in normal gravity the highest rate of fuel reaction (consumption) occurs close to the base of the flame. In microgravity, the highest reactivity occurs at the top and dimishes toward the base.

5. Interactions between flames could be enhanced in microgravity. Usually the height-to-width ratio is lower in microgravity; the flames have a tendency to be thicker. There is a critical distance between two parallel burning surfaces where the interaction is most enhanced. At shorter distances the flame strength in the inside dimishes, while at increased distance the interaction dimishes until there are two separate flames. The range of separation distance where there is flame interaction is larger in microgravity. For paper in normal gravity the flames were observed to be separated at $10 \mathrm{~mm}$, while it was observed that in microgravity at $40 \mathrm{~mm}$ distance the flames were still connected (NASA Glenn).

6. Flame oscillations were observed forming both in microgravity and normal gravity at near extinction when the oxygen is being depleted, with the result of the flame base extinguishing. Since the material is still hot, fuel still vaporizes and forms with oxygen a combustible mixture, so a flashback of the flame base can occur. This further depletes the oxygen so that more of the 
flame base extinguishes, and the cycle repeats. The oscillations continue with increasing amplitude, until the ambient oxygen concentration becomes too low to sustain any part of the flame.

\section{Slide 6}

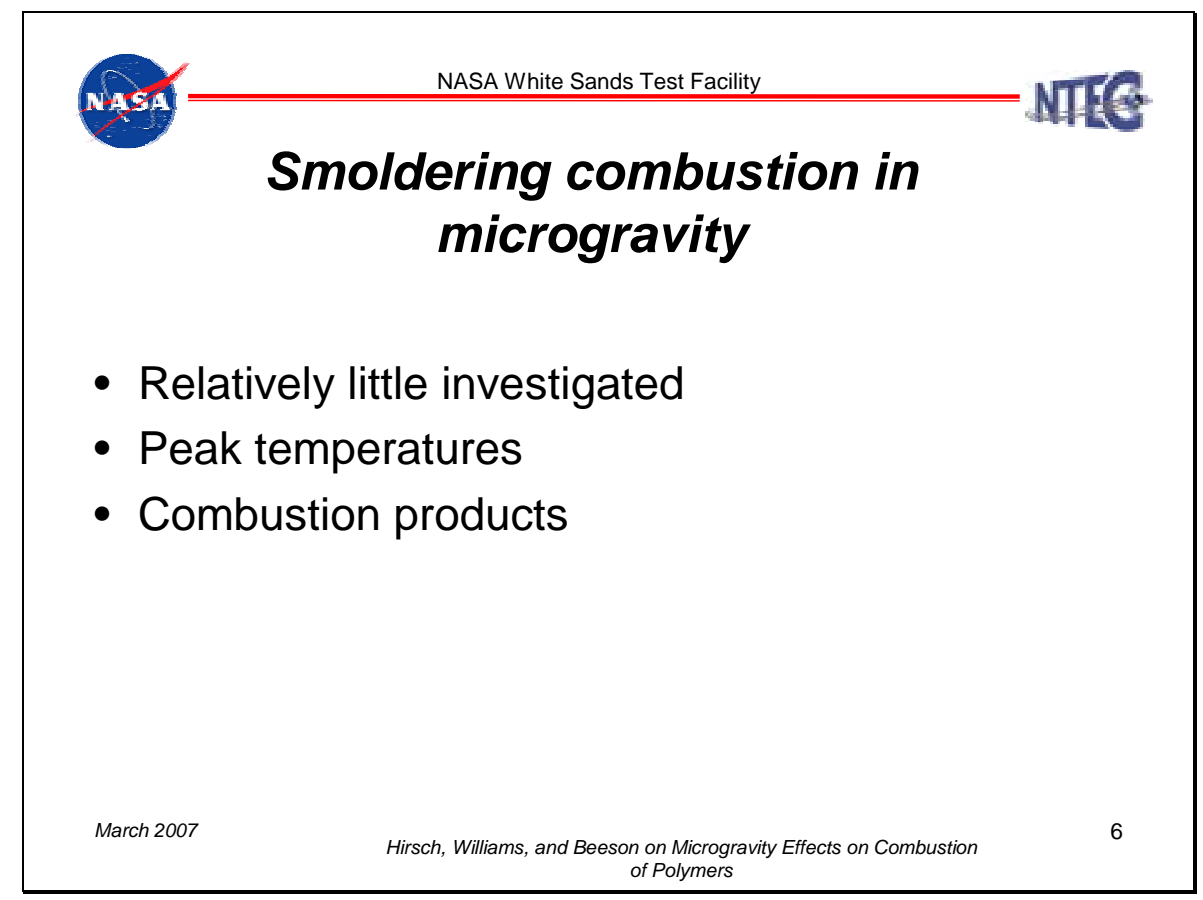

7. Smoldering combustion is a solid-phase combustion of porous materials. Smoldering reaction and propagation rates are determined by the balance between the transport of oxygen to the reaction zone and the transport of energy to and from the reaction zone. When heat losses are significant compared with the heat produced, the rate of heat loss is controlling and the smoldering is weak. Generally, the oxygen transport is controlling smoldering which is more vigorous. Smoldering can propagate slowly, undetected for long periods of time, and suddenly undergo transition to flaming. In microgravity, the peak temperatures measured were slightly higher than in normal gravity (Jose Torero, Fernandez-Pello, University of California at Berkeley), presumably due to buoyant cooling in normal gravity. Never-the-less, it was observed that the amount of carbon monoxide produced in microgravity was much larger than in normal gravity. 
Slide 7

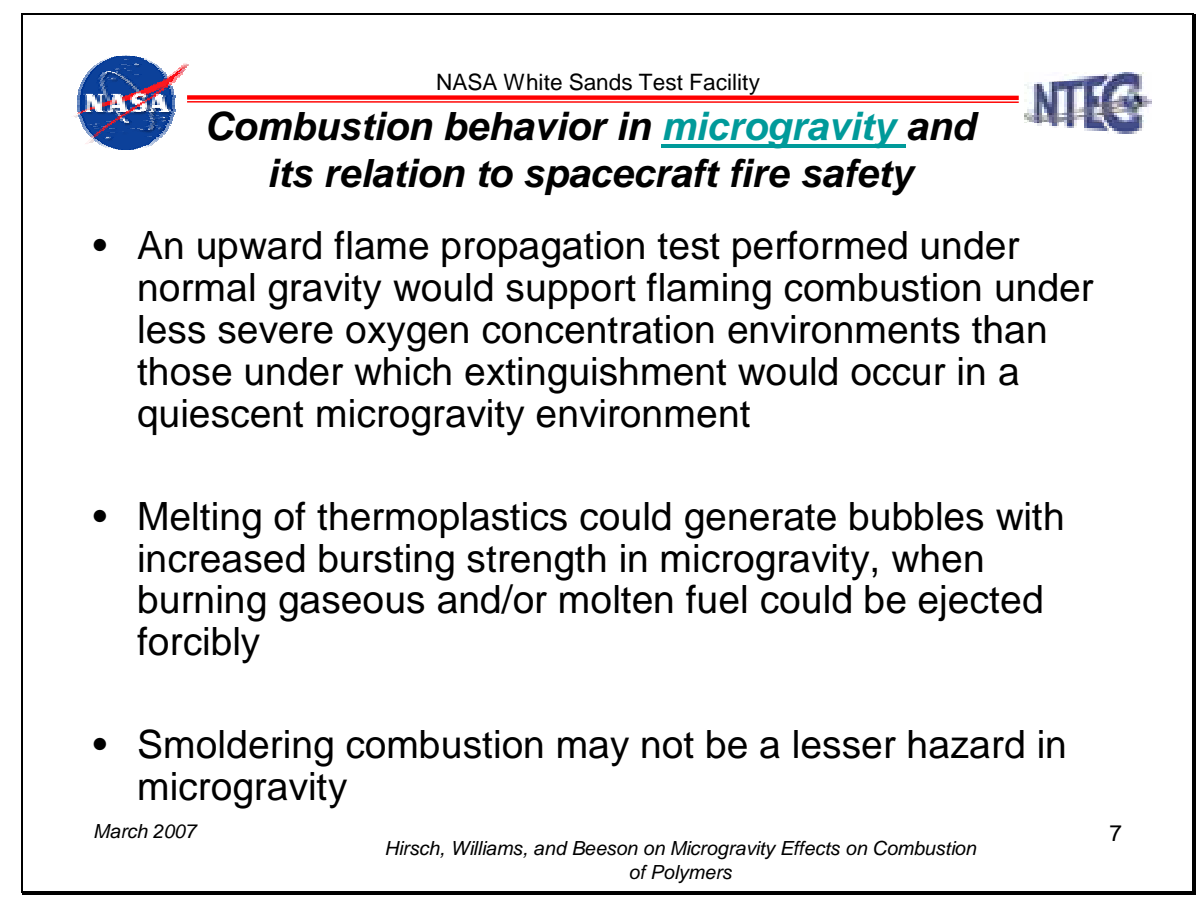

No notes for this slide. 